\title{
Control Synthesis for Non-Polynomial Systems: A Domain of Attraction Perspective
}

\author{
Dongkun Han and Matthias Althoff
}

\begin{abstract}
This paper studies a control synthesis problem to enlarge the domain of attraction (DA) for non-polynomial systems by using polynomial Lyapunov functions. The basic idea is to formulate an uncertain polynomial system with parameter ranges obtained from the truncated Taylor expansion and the parameterizable remainder of the non-polynomial system. A strategy for searching a polynomial output feedback controller and estimating the lower bound of the largest DA is proposed via an optimization of linear matrix inequalities (LMIs). Furthermore, in order to check the tightness of the lower bound of the largest estimated DA, a necessary and sufficient condition is given for the proposed controller. Lastly, several methods are provided to show how the proposed strategy can be extended to the case of variable Lyapunov functions. The effectiveness of this approach is demonstrated by numerical examples.
\end{abstract}

\section{INTRODUCTION}

Estimating the domain of attraction (DA) of an equilibrium-point is a key issue in the area of systems and control engineering. The sublevel sets of Lyapunov functions are proven to be an effective way, for providing an inner estimate of the DA [1]. Due to the developments in positive polynomials, a number of mature results have been obtained in the estimation of the DA for polynomial systems [2]-[5]. Optimization methods involving linear matrix inequalities (LMIs) and semidefinite programming have been proposed using the sum of squares (SOS) technique and the square matrix representation (SMR) [6]-[9].

However, emerging phenomena in various areas such as aerospace engineering and system biology demand for solutions involving non-polynomial models. In order to deal with this kind of model, one useful approach is to approximate the nonlinear function by a polynomial one and use the existing methods for polynomial systems [10]-[12]. In [10], the nonlinear function is approximated by a sum of a group of polynomial functions, non-polynomial functions, and the products of both. Then, the non-polynomial functions are replaced by new state variables. Using the same model, a novel method is proposed that the non-polynomial function is over-approximated by polynomials via Taylor expansion [13]. In [11], the non-polynomial function is assumed to be in the convex hull of a set of polynomial functions, and a stability condition is proposed by using a generalized linear differential inclusions. In [12], the state space is recast into a higher dimensional one by introducing new variables to replace the non-polynomial terms of the original system. On

The authors are with the Institut für Informatik, Technische Universität München, Boltzmannstraße 3, 85748 Garching bei, München, Germany. E-mail: dongkun.han@tum.de. the other hand, there are some effective approaches which investigate the DA of non-polynomial systems without polynomial approximation [14], [15]. In [14], the nonlinear systems is transformed to a linear system with state-dependent input saturation. In this work, a novel approach is provided by using exact feedback linearization. In [15], based on the multidimensional gridding approach, a strategy is proposed for estimating the DA by employing Chebychev points and a fixed quadratic Lyapunov function. For non-polynomial systems, these explorations are useful for estimating the DA. However, the control synthesis problem for non-polynomial systems is rarely considered. In contrast to the literature mentioned above, this paper gives a strategy for searching an output feedback controller and estimating the lower bound of the largest estimated DA.

The main contributions of this paper are threefold:

- For the first time, an approach is proposed for computing a static output feedback controller, which maximizes the largest estimate of the DA for non-polynomial systems with the truncated Taylor expansion and the parameterizable remainder.

- By exploiting SMR and a transformation of a power vector, a lower bound of the largest estimate of the DA can be computed by solving a generalized eigenvalue problem.

- An asymptotic non-conservative condition is given for the lower bound of the largest estimate of the DA with a selected Lyapunov function, and a necessary and sufficient condition is also provided for establishing the tightness of this lower bound, based on the computed controller.

\section{PREliminaries}

Notations: $\mathbb{N}, \mathbb{R}$ : natural and real number sets; $\mathbb{R}_{+}$: positive real number set; $0_{n}$ : origin of $\mathbb{R}^{n} ; \mathbb{R}_{0}^{n}: \mathbb{R}^{n} \backslash\left\{0_{n}\right\} ; A^{T}$ : transpose of $A ; I_{n}: n \times n$ identity matrix; $A>0(A \geq 0)$ : symmetric positive definite (semidefinite) matrix $A ; A \otimes B$ : Kronecker product of matrices $A$ and $B ; \operatorname{tr}(A)$ : trace of matrix $A ; \operatorname{ker}(A)$ : null space of linear map $A ; \operatorname{ver}(\mathscr{P})$ : set of vertices of the polytope $\mathscr{P} ; \operatorname{co}\left\{X_{1}, \ldots, X_{p}\right\}$ : convex hull of matrices $X_{1}, \ldots, X_{p} \in \mathbb{R}^{m \times n} ; \operatorname{deg}(f)$ : degree of polynomial function $f$; $\operatorname{diag}(v)$ : a square diagonal matrix with the elements of vector $v$ on the main diagonal; $(*)^{T} A B$ in a form of SMR: $B^{T} A B$. Let $\mathcal{P}$ be the set of polynomials and $\mathcal{P}^{n \times m}$ be the set of matrix polynomial with dimension $n \times m$. A polynomial $p(x) \in \mathcal{P}$ is nonnegative if $p(x) \geq 0$ for all $x \in \mathbb{R}^{n}$. An effective way of checking whether $p(x)$ is 
positive consists of checking whether $p(x)$ can be expressed as a sum of squares of polynomials (SOS), i.e.,

$$
p(x)=\sum_{i=1}^{k} p_{i}(x)^{2}
$$

for some $p_{1}, \ldots, p_{k} \in \mathcal{P}$. Obviously, any SOS polynomial is nonnegative. We denote the set of SOS polynomials as $\mathcal{P}^{\mathrm{SOS}}$. If $p(x) \in \mathcal{P}^{\mathrm{SOS}}$ becomes 0 only for $x=0_{n}$, we call $p(x)$ local SOS which is denoted by $\mathcal{P}_{0}^{\text {SOS }}$.

\section{A. Model Formulation}

In this paper, we consider the following model:

$$
\begin{aligned}
& \dot{x}(t)=f(x(t))+\sum_{i=1}^{r} g_{i}(x(t)) \zeta_{i}\left(x_{a_{i}}(t)\right)+W(x(t)) u(t) \\
& y(t)=h(x(t)), x(0)=x_{\text {init }}
\end{aligned}
$$

using the state vector $x \in \mathbb{R}^{n}$, the initial state $x_{\text {init }} \in \mathbb{R}^{n}$, the polynomial functions $f \in \mathcal{P}^{n}, g_{1}, \ldots, g_{r} \in \mathcal{P}, h \in$ $\mathcal{P}^{n_{y}}, W \in \mathcal{P}^{n \times n_{u}}$, the non-polynomial functions $\zeta_{1}, \ldots, \zeta_{r}$ : $\mathbb{R} \rightarrow \mathbb{R}^{n}$ and the indexes $a_{1}, \ldots, a_{r} \in\{1, \ldots, n\}$. In this paper, we consider that the control input $u(t) \in \mathbb{R}^{n_{u}}$ is in the form of

$$
u(t)=U(y(t))=K \phi\left(n_{y}, d_{u}\right),
$$

where $U \in \mathcal{P}^{n_{u}}, d_{u}$ is the degree of $U(y(t))$ in $y, K$ is a constant matrix, $\phi\left(n_{y}, d_{u}\right)$ is a power vector containing all the monomials of degree less or equal to $d_{u}$, which will be introduced in detail in Section III. In the sequel, we will omit the arguments $t$ and $x$ of functions whenever possible for the brevity of notations.

Remark 1: Without loss of generality, we set the origin as the equilibrium-point of interest, while the origin is not necessarily stable for $u \equiv 0_{n_{u}}$.

In order to make this model more practical, the control input is supposed to be finite and $K$ belongs to an arbitrary hyper-rectangle:

$\mathcal{K}=\left\{K: K_{i j} \in\left[\underline{K}_{i j}, \bar{K}_{i j}\right], i=1, \ldots, n_{u}, j=1, \ldots, n_{r}\right\}$. In this work, we consider that the non-polynomial functions $\zeta_{i}, i=1, \ldots, r$, are $k$ times differentiable at point 0 and $k+1$ times differentiable on the open interval between 0 and $x_{a_{i}}$, then $\zeta_{i}$ can be rewritten in the form of a Maclaurin polynomial, i.e., Taylor expansion evaluated at point 0 , as follows:

$$
\zeta_{i}\left(x_{a_{i}}\right)=\eta_{i}\left(x_{a_{i}}\right)+\xi_{i} \frac{x_{a_{i}}^{k+1}}{(k+1) !}
$$

where $\xi_{i} \in \mathbb{R}$ is a parameter which can be appropriately chosen and $\eta_{i}\left(x_{a_{i}}\right)$ is the $k$-th order Taylor polynomial:

$$
\eta_{i}\left(x_{a_{i}}\right)=\left.\sum_{j=0}^{k} \frac{d^{j} \zeta_{i}\left(x_{a_{i}}\right)}{d x_{a_{i}}^{j}}\right|_{x_{a_{i}}=0} \frac{x_{a_{i}}^{j}}{j !} .
$$

Remark 2: The residual, or also called Taylor remainder, $\zeta_{i}\left(x_{a_{i}}\right)-\eta_{i}\left(x_{a_{i}}\right)$ is parameterized by $\xi_{i}$, which is constrained in the rectangle

$$
\Xi=\left[\underline{\tau}_{1}, \bar{\tau}_{1}\right] \times \cdots \times\left[\underline{\tau}_{r}, \bar{\tau}_{r}\right]
$$

and $\underline{\tau}_{i}, \bar{\tau}_{i} \in \mathbb{R}, i=1, \ldots, r$, are selected as the tightest bounds satisfying

$$
\underline{\tau}_{i} \leq\left.\frac{d^{k+1} \zeta_{i}\left(x_{a_{i}}\right)}{d x_{a_{i}}^{k+1}}\right|_{x_{a_{i}=\iota}} \leq \bar{\tau}_{i}
$$

for all $\iota \in \mathcal{I}$, where $\mathcal{I}$ is the set of interest, which is chosen in the sublevel set of a Lyapunov function $\mathcal{V}_{x_{a_{i}}}$ that we will introduce later. Note that the residual can also be expressed in other mean-value forms, such as the Cauchy form, which also has the property (7). For more details about the Taylor expansion, please find [16] and references therein.

\section{B. Problem Formulation}

First, let us introduce the DA of the origin. It is the set of initial states for which the system asymptotically converges to the origin, which can be expressed by

$$
\mathcal{D}=\left\{x_{\text {init }} \in \mathbb{R}^{n}: \lim _{t \rightarrow+\infty} \chi\left(t ; x_{\text {init }}, K\right)=0_{n}\right\},
$$

where $\chi$ is the solution of system (2). In this paper, we aim to design a controller (3) to enlarge the estimate of the DA of the origin via polynomial Lyapunov functions. Specifically, let $v(x)$ be a Lyapunov function of system (2) for the origin, which satisfies

$$
\forall x \in \mathbb{R}_{0}^{n}: v(x)>0, v\left(0_{n}\right)=0, \lim _{\|x\| \rightarrow \infty} v(x)=\infty
$$

and the time derivative of $v(x)$ along the trajectories of (2) is locally negative definite [1]. To this end, we introduce the sublevel set of $v(x)$ as

$$
\mathcal{V}(c)=\left\{x \in \mathbb{R}^{n}: v(x) \leq c\right\}
$$

where $c \in \mathbb{R}^{+}$. For system (2), $\mathcal{V}$ is an estimate of $\mathcal{D}$ if there exists a $K \in \mathcal{K}$ such that

$$
\forall x \in \mathcal{V}(c) \backslash\{0\}, \forall \xi_{i} \in \Xi: \dot{v}\left(x, K, \xi_{i}\right)<0 .
$$

Let us propose the main problems we are concerned with: find an optimal matrix $K$ such that the estimate of the DA is maximized under certain selected criteria, i.e., solving

$$
\begin{aligned}
& \mu=\sup _{c, v, K} \rho(\mathcal{V}(c)) \\
& \text { s.t. }\left\{\begin{array}{l}
(8)-(10) \text { hold } \\
K \in \mathcal{K} \\
\xi_{i} \in \Xi, \forall i=1, \ldots, r
\end{array}\right.
\end{aligned}
$$

where $\rho$ is a measure of $\mathcal{V}(c)$ depicting the selected userdefined criteria. It is worth noting that problem (11) can be relaxed to a trilinear matrix inequality problem, which in nature is non-convex [17]. In order to solve this problem, iteration algorithms and sub-optimization problems are proposed [18]. Specifically, with regard to the problem (11), we start with a selected polynomial Lyapunov function (i.e., (8) holds), and find an admissible controller $K$ such that the estimate of $\mathcal{V}(c)$ is maximized, i.e., we aim at finding

$$
\gamma=\sup _{c, K} c
$$

for all $K \in \mathcal{K}$ and $\xi_{i} \in \Xi, i=1, \ldots, r$, such that (10) holds. 


\section{MAin Results}

In this section, we first propose an approach to compute the lower bound of the largest estimate of the DA based on a fixed Lyapunov function. Then, we extend our results to the case of variable Lyapunov functions.

\section{A. Fixed Lyapunov Function}

Real Nullstellensatz (N-satz) and Positivestellensatz (Psatz) are fundamental techniques to establish the nonnegativity over a semialgebraic set, which is described by polynomial inequalities. For more details, please find [19]-[21] and references therein. A stronger version of the P-satz for the case of basic compact semialgebraic sets is given as follows:

Lemma 1: ([22]) Let $f_{1}, \ldots, f_{n}$ be polynomials of even degree such that the set

$$
\mathcal{F}=\left\{x \in \mathbb{R}^{n}: f_{1}(x) \geq 0, \ldots, f_{m}(x) \geq 0\right\}
$$

is compact and the highest degree forms have no common zeros in $\mathbb{R}_{0}^{n}$, then there is a polynomial $p$ such that

$$
\begin{aligned}
\forall x \in \mathcal{F}: p(x)>0, \quad \Longleftrightarrow \\
\quad \exists s_{0}, \ldots, s_{m} \in \mathcal{P}^{\mathrm{SOS}}: p=s_{0}+\sum_{i=1}^{k} s_{i} f_{i} .
\end{aligned}
$$

This lemma provides an essential perspective that any strictly positive polynomial $p(x) \in \mathcal{F}$ is actually in the cone generated by $f_{i}$. Based on this, one can derive a result by exploiting the local SOS cone, which builds a bridge between the estimate of the DA and SOS programming.

Lemma 2: For polynomials $a_{1}, \ldots, a_{n}, b_{1}, \ldots, b_{l}$ and $p$, define a set

$$
\begin{aligned}
\mathcal{B}= & \left\{x \in \mathbb{R}^{n}: a_{i}(x)=0, \forall i=1, \ldots, m,\right. \\
& \left.b_{i}(x) \geq 0, \forall j=1, \ldots, l\right\} .
\end{aligned}
$$

Let $\mathcal{B}$ be compact. Condition $\forall x \in \mathcal{B}: p(x)>0$ can be established if the following condition holds:

$$
\left\{\begin{array}{l}
\exists r_{1}, \ldots, r_{m} \in \mathcal{P}, s_{1}, \ldots, s_{l} \in \mathcal{P}_{0}^{\mathrm{SOS}} \\
p-\sum_{i=1}^{m} r_{i} a_{i}-\sum_{i=1}^{l} s_{i} b_{i} \in \mathcal{P}_{0}^{\mathrm{SOS}}
\end{array}\right.
$$

Proof: Let $s_{0} \in \mathcal{P}_{0}^{\mathrm{SOS}}$, this result can be obtained from Lemma 1 by setting

$$
p=\sum_{i=1}^{m} r_{i} a_{i}+\sum_{i=1}^{l} s_{i} b_{i}+s_{0} .
$$

Remark 3: By using the local SOS cone, condition (16) provides a sufficient condition to establish the positivity of a polynomial on a compact set. Condition (16) is also a necessary condition if the auxiliary local SOS polynomial $s_{i}$ has unbounded degree and there is a polynomial $b$ in set $\mathcal{B}$ such that $b^{-1}[0, \infty)$ is compact in $\mathbb{R}^{n}$. For details please refer to [22] for the case of SOS cones.
Denote $\xi=\left(\xi_{1}, \ldots, \xi_{r}\right)^{T}$ and for the brevity of presentation, let us introduce the following polynomials:

$$
\begin{aligned}
& r(x)=\frac{\partial v(x)}{\partial x}\left(f(x)+\sum_{i=1}^{r} g_{i}(x) \eta_{i}\left(x_{a_{i}}\right)\right) \\
& q_{i}(x)=\frac{\partial v(x)}{\partial x} g_{i}(x) \frac{x_{a_{i}}^{(k+1)}}{(k+1) !} \\
& q(x)=\left(q_{1}(x), \ldots, q_{r}(x)\right)^{T} \\
& e(x, K)=\frac{\partial v(x)}{\partial x} W(x) U(K, x) .
\end{aligned}
$$

Based on the P-satz and its extensions above, an approach is provided to compute a lower bound of $\gamma$ in (12).

Theorem 1: Define $k$ as the largest order of the Maclaurin polynomial in (4). Provided that there exists a polynomial $s(x) \in \mathcal{P}_{0}^{\mathrm{SOS}}$ and $c_{k}$ is the optimum of the following polynomial optimization

$$
\begin{aligned}
& c_{k}=\sup _{c, K, s} c \\
& \text { s.t. }\left\{\begin{array}{l}
-\psi(x, K, c, s(x)) \in \mathcal{P}_{0}^{\mathrm{SOS}} \\
\forall x \in \mathcal{V}(c) \backslash\{0\} \\
\forall \xi_{i} \in \operatorname{ver}(\Xi), i=1, \ldots, r,
\end{array}\right.
\end{aligned}
$$

where

$$
\begin{aligned}
\psi(x, K, c, s(x))= & r(x)+q(x)^{T} \xi \\
& +e(x, K)+s(x)(c-v(x)),
\end{aligned}
$$

functions $r, q$ and $e$ are introduced by (18)-(21), a local SOS polynomial $s(x) \in \mathcal{P}_{0}^{\mathrm{SOS}}$ and $\operatorname{ver}(\Xi)$ is the set of vertices of $\Xi$. Then, $c_{k} \leq \gamma$.

Proof: $c_{k}$ is a lower bound of $\gamma$ if under condition (22), $\mathcal{V}\left(c_{k}\right)$ is an under-estimate of the DA of the origin for system (2), i.e., there exists a $K \in \mathcal{K}$ such that (10) holds. Thus, our main effort in this proof is to derive (10) from (22).

Suppose (22) holds, one has that $-\psi(x, K, c, s(x))$ and $s(x)$ are local SOS. From Lemma 2, it yields that

$$
\forall \xi_{i} \in \operatorname{ver}(\Xi): r(x)+q(x)^{T} \xi+e(x, K)<0,
$$

for all $x$ in $\left\{x \in \mathbb{R}^{n}: c-v(x) \geq 0\right\} \backslash\{0\}$. From (4) and (7), one has that for all $x \in \mathcal{V}(c)$, there exists $\bar{\xi}_{i} \in\left[\underline{\tau}_{i}, \bar{\tau}_{i}\right]$ such that $\zeta_{i}$ in (4) can be expressed as

$$
\zeta_{i}\left(x_{a_{i}}\right)=\eta_{i}\left(x_{a_{i}}\right)+\bar{\xi}_{i} \frac{x_{a_{i}}^{(k+1)}}{(k+1) !} .
$$

Thus, from (18)-(21) one obtains that $\forall x \in \mathcal{V}(c)$ : there exist $\bar{\xi}_{i} \in \Xi$ for all $i=1, \ldots, r$ and $\bar{\xi}=\left(\bar{\xi}_{1}, \ldots, \bar{\xi}_{r}\right)^{T}$ such that

$$
\dot{v}(x, K, \bar{\xi})=r(x)+q(x)^{T} \bar{\xi}+e(x, K) .
$$

Since $\dot{v}(x, K, \bar{\xi})$ is affine in $\bar{\xi}$ while $\Xi$ is a convex polytope, from (24)-(26), one has that there exists a $K \in \mathcal{K}$ such that

$$
r(x)+q(x)^{T} \bar{\xi}+e(x, K)<0
$$

for all $\bar{\xi}_{i} \in \Xi$ and for all $x$ in $\left\{x \in \mathbb{R}^{n}: c-v(x) \geq\right.$ $0\} \backslash\{0\}$, i.e., (10) holds. Therefore, $\mathcal{V}\left(c_{k}\right)$ is an estimate of the DA with the truncation degree $k$. Taking into account the definition of $\gamma$ in (12), it finally yields that $c_{k}$ is a lower bound of $\gamma$, which completes this proof. 
Remark 4: For this result, note that

- Theorem 1 provides an optimization method consisting of SOS programming to compute a guaranteed lower bound $c_{k}$ of the sought $\gamma$. This method belongs to an inner estimation (under-approximation) approach by using the set $\mathcal{V}\left(c_{k}\right)$ corresponding to a chosen truncation order $k$.

- The non-polynomial function $\zeta_{i}$ is expressed by Taylor expansion with a parameterized remainder in Lagrange form where the parameters $\xi_{i}$ are constrained in a polytope, making the remainder considered in [10] a special case.

\section{B. Bound Computation by Square Matrix Representation} (SMR)

Observe that the condition of Theorem 1 is not easy to establish since searching for a local SOS $s(x)$ and a scalar $c$ at the same time makes this problem non-convex. In order to cope with this issue, a new class of SMR is introduced for polynomials belonging to the set of local SOS, i.e., $p_{0}(x) \in$ $\mathcal{P}_{0}^{\text {SOS }}$, and an approach is provided by solving a generalized eigenvalue problem for computing the lower bound of $\gamma$.

First we will introduce a new class of SMR for the set of local SOS. Consider a polynomial $p_{0}(x)$ of degree $2 d_{x}$ without the constant and linear terms, one has $p_{0}(x) \in \mathcal{P}_{0}^{\text {SOS }}$. For this sort of polynomial, the SMR can be given as follows:

$$
p_{0}(x)=(*)^{T}(P+L(\delta)) \phi\left(n, d_{x}\right)
$$

where $(*)^{T} A B$ is short for $B^{T} A B$ introduced in Section II, $P$ is denoted by the SMR matrix of $p_{0}(x), n$ is the number of variables, $\phi\left(n, d_{x}\right) \in \mathbb{R}^{l\left(n, d_{x}\right)}$ is called the power vector containing all monomials of degree less or equal to $d_{x}$ without degree $0, L(\delta)$ is a parameterization of the affine space

$$
\begin{aligned}
\mathscr{L}= & \left\{L(\delta) \in \mathbb{R}^{\phi \times \phi}: L(\delta)=L^{T}(\delta),\right. \\
& \left.(*)^{T} L(\delta) \phi\left(n, d_{x}\right)=0\right\}
\end{aligned}
$$

in which $\delta \in \mathbb{R}^{\vartheta\left(n, d_{x}\right)}$ is a vector of free parameters. The dimensions of $\phi \in \mathbb{R}^{l\left(n, d_{x}\right)}$ and $\delta \in \mathbb{R}^{\vartheta\left(n, d_{x}\right)}$ can be calculated analogously to [4]. An exemplary illustration of SMR is provided as follows.

Given the polynomial $f(x)=3 x^{6}+2 x^{5}+4 x^{4}+5 x^{2}$, we have $d_{x}=3, n=1$ and $\phi\left(n, d_{x}\right)=\left(x^{3}, x^{2}, x\right)^{T}$. Then, $f(x)$ can be written as follows,

$$
P=\left(\begin{array}{ccc}
3 & 1 & 0 \\
1 & 4 & 0 \\
0 & 0 & 5
\end{array}\right), L(\delta)=\left(\begin{array}{ccc}
0 & 0 & -\delta \\
0 & 2 \delta & 0 \\
-\delta & 0 & 0
\end{array}\right)
$$

Let $2 d_{v}, 2 d_{d}$ and $2 d_{s}$ be the degree of $v(x), \dot{v}(x, K)$ and $s(x)$ respectively which are introduced in (23) and (26), and $d_{d}-d_{v} \leq d_{s}$. Observe the constant and linear terms of $v(x)$, $s(x)$ and $\psi(x)$ are null, let $d_{\psi}=d_{v}+d_{s}$, by exploiting the representation introduced in (28), we have the following SMR:

$$
\begin{aligned}
v(x) & =(*)^{T} V \phi\left(n, d_{v}\right) \\
s(x) & =(*)^{T} S \phi\left(n, d_{s}\right) \\
\psi(x) & =(*)^{T} \Psi(\delta, K, c, \xi, S) \phi\left(n, d_{\psi}\right)
\end{aligned}
$$

where $\delta \in \mathbb{R}^{\vartheta\left(n, d_{\psi}\right)}$ is a vector of free parameters, $V \in$ $\mathbb{R}^{l\left(n, d_{v}\right) \times l\left(n, d_{v}\right)}, S \in \mathbb{R}^{l\left(n, d_{s}\right) \times l\left(n, d_{s}\right)}$ and $\Psi(\delta, K, c, \xi, S) \in$ $\mathbb{R}^{l\left(n, d_{\psi}\right) \times l\left(n, d_{\psi}\right)}$ are symmetric matrices. Let $R(\delta), Q(\xi)$, $E(K), \Lambda_{1}(S)$ and $\Lambda_{2}(S)$ be SMR matrices of $r(x), q(x)^{T} \xi$, $e(x, K), s(x)$ and $v(x) s(x)$, respectively. From (23), it is clear that

$\Psi(\delta, K, c, \xi, S)=R(\delta)+Q(\xi)+E(K)+c \Lambda_{1}(S)-\Lambda_{2}(S)$

where $\delta \in \mathbb{R}^{\vartheta\left(n, d_{\psi}\right)}$ is a vector of free parameters. Based on the SMR introduced above, a result can be obtained directly from Theorem 1 .

Lemma 3: $\tilde{\gamma}$ is a lower bound of $\gamma$ if $\tilde{\gamma}=\sup \left\{c_{k}\right\}$ and there exist $S>0, K \in \mathcal{K}$ and for all $\xi_{i} \in \operatorname{ver}(\Xi), i=$ $1, \ldots, r$, such that $\Psi(\delta, K, c, \xi, S)<0$.

Proof: Notice that $\Psi(\delta, K, c, \xi, S)$ and $S$ are the SMR matrices of $\psi(\delta, K, c, \xi, S)$ and $s(x)$ respectively, if there exist a $S>0$ and a $K \in \mathcal{K}$ such that $\Psi(\delta, K, c, \xi, S)<0$ for all $\xi_{i} \in \operatorname{ver}(\Xi)$, one has that $-\psi(\delta, K, c, S) \in \mathcal{P}_{0}^{\text {SOS }}$ and $s(x) \in \mathcal{P}_{0}^{\text {SOS }}$. From Theorem 1 , it yields that $\tilde{\gamma} \leq \gamma$, which ends this proof.

Let us observe that the scalar $c$ and $S \in \mathbb{R}^{l\left(n, d_{s}\right) \times l\left(n, d_{s}\right)}$ are in a bilinear relationship, which makes this problem non-convex. In order to handle this problem in polynomial time, a transformation of the power vector is introduced and based on this transformation, an optimization consisting of a generalized eigenvalue problem is formulated.

Lemma 4: Let $\varphi_{1}$ and $\varphi_{2}$ be given positive scalars, and $d_{\psi}=d_{v}+d_{s}$. Then, the polynomial $\varsigma(x)=\varphi_{1} s(x)+$ $\varphi_{2} v(x) s(x)$ can be expressed in the following SMR form

$$
\varsigma(x)=(*)^{T} \Lambda(S) \phi\left(n, d_{\psi}\right)
$$

where $V, S$ are SMR matrices introduced by (30) and (31), $\Lambda(S)$ can be expressed by

$$
\Lambda(S)=(*)^{T}\left(\left[\begin{array}{cc}
\varphi_{1} & 0 \\
0 & \varphi_{2} V
\end{array}\right] \otimes S\right) H
$$

and matrix $H$ satisfies

$$
H \phi\left(n, d_{\psi}\right)=\left[\begin{array}{c}
1 \\
\phi\left(n, d_{v}\right)
\end{array}\right] \otimes \phi\left(n, d_{s}\right) .
$$

Proof: See Appendix VII-A.

Based on the transformation provided by Lemma 4, the main result of this paper can be proposed now.

Theorem 2: Consider a fixed Lyapunov function $v(x)=$ $(*)^{T} V \phi\left(n, d_{v}\right)$ and given positive scalars $\varphi_{1}, \varphi_{2}$. The lower bound of $\tilde{\gamma}$ can be computed by

$$
\tilde{\gamma}=-\frac{\tilde{e}}{\varphi_{1}+\varphi_{2} \tilde{e}}
$$

where $\tilde{e}$ is the solution of the following generalized eigenvalue problem

$$
\begin{aligned}
& \tilde{e}=\inf _{K, S, \delta, e} e \\
& \text { s.t. }\left\{\begin{array}{l}
\varphi_{1}+\varphi_{2} e>0 \\
S>0 \\
e \Lambda(S)>R(\delta)+Q(\xi)+E(K)-\Lambda_{2}(S) \\
K \in \mathcal{K}, \xi_{i} \in \operatorname{ver}(\Xi), \forall i=1, \ldots, r .
\end{array}\right.
\end{aligned}
$$


Proof: From the third inequality of (36), one has

$$
\begin{aligned}
\widetilde{\Phi}(\delta, K, e, \xi, S)= & R(\delta)+Q(\xi)+E(K) \\
& -e \Lambda(S)-\Lambda_{2}(S) \\
< & 0, \forall \xi_{i} \in \Xi .
\end{aligned}
$$

Observe $\widetilde{\Phi}(\delta, K, e, \xi, S)$ is the SMR matrix of the polynomial

$$
\begin{aligned}
\tilde{\psi}(x, K, e, \xi, s(x))= & \dot{v}(x, K, \xi)-v(x) s(x) \\
& -e\left(\varphi_{1}+\varphi_{2} v(x)\right) s(x),
\end{aligned}
$$

we rewrite $\tilde{\phi}(x, K, e, \xi, s(x))$ in the following form:

$$
\tilde{\psi}(x, K, e, \xi, s(x))=\tilde{\psi}\left(x, K, \xi, \frac{-e}{\varphi_{1}+\varphi_{2} e},\left(\varphi_{1}+\varphi_{2} e\right) s(x)\right) .
$$

Notice that for all $e \in\left(-\left(\varphi_{1} / \varphi_{2}\right), 0\right]$, the function $-e /\left(\varphi_{1}+\right.$ $\left.\varphi_{2} e\right)$ is monotonically decreasing and its corresponding mapping range is the interval $[0,+\infty)$, it directly yields that the lower bound of $\tilde{\gamma}$ can be calculated by (35).

Next, let us prove $\Lambda(S)$ is positive definite which makes (36) a generalized eigenvalue problem. Observe that the matrix dimensions of $\Lambda, S$ and $V$ satisfying

$$
l\left(n, d_{\psi}\right)<l\left(n, d_{s}\right)\left(l\left(n, d_{v}\right)\right),
$$

which means that a shrunk SMR matrix is obtained after the power vector transformation in (34). Moreover, any monomial of the power vector $\phi\left(x, d_{\psi}\right)$ is included in the monomial set of the power vector $\left[1, \phi\left(n, d_{v}\right)\right]^{T} \otimes \phi\left(n, d_{s}\right)$, which directly implies that matrix $H$ has full rank. From Lemma 4, one has $\Lambda>0$ if $V>0$ and $S>0$, which completes this proof.

For details of the definition and the construction of the generalized eigenvalue problem, please find [23].

\section{Tightness Investigation}

The last theorem gives a useful strategy to compute a guaranteed lower bound of $\tilde{\gamma}$. Another question arises naturally: Does the computed $\tilde{\gamma}$ asymptotically converge to the desired $\gamma$ ? This subsection provides an answer with nonconservatism.

Proposition 1: Assume $\gamma<+\infty$ and there exists a positive scalar $\theta \in \mathbb{R}, \theta<+\infty$ satisfying

$$
\theta=\max \left\{\left|\underline{\tau}_{1}\right|,\left|\bar{\tau}_{1}\right|, \ldots,\left|\underline{\tau}_{r}\right|,\left|\bar{\tau}_{r}\right|\right\}, \forall c \leq \gamma .
$$

where $k$ is the order of the Maclaurin polynomial in (4). Then, $\lim _{k \rightarrow \infty} c_{k}=\gamma$.

Proof: See Appendix VII-B.

Proposition 1 gives an asymptotic non-conservatism criteria for computing the lower bound $c_{k}$. However, condition $k \rightarrow \infty$ is not always practical because setting a large value of the truncation degree $k$ may lead to a high computation complexity. Naturally one may ask: for a given finite number $k$, is there any condition to establish the tightness of $\gamma$ ? The following theorem is proposed to answer this question.

Theorem 3: Let $\bar{K}, \bar{S}, \bar{\delta}$ and $\bar{\xi}$ be the optimal values of $K, S, \delta$ and $\xi$ in the optimization (36), and define

$$
\begin{aligned}
\bar{\Gamma}(\bar{\delta}, \bar{\xi}, \bar{K}, \bar{S})= & R(\bar{\delta})+Q(\bar{\xi}, \bar{\tau}) \\
& +E(\bar{K})-\Lambda_{2}(\bar{S})-\tilde{e} \Lambda(\bar{S})
\end{aligned}
$$

Then, a necessary and sufficient condition for $\tilde{\gamma}=\gamma$ is that there exists a $\bar{x} \in \mathcal{T}$ where

$$
\mathcal{T}=\left\{\bar{x} \in \mathbb{R}^{n}: \phi\left(n_{\bar{x}}, d_{\psi}\right) \in \operatorname{ker}(\bar{\Gamma}) \text { and } \dot{v}(\bar{x}, \bar{\xi}, \bar{K})=0\right\} .
$$

Proof: (Sufficiency) Let us recall that the largest estimate of the DA can be obtained by $\mathcal{V}(\gamma)$ where

$$
\gamma=\sup _{x \in \mathbb{R}_{0}^{n}, K \in \mathcal{K}} v(x) \text { s.t. } \dot{v}(x, \xi, K)=0 .
$$

Assume that $\tilde{\gamma}=\gamma(\bar{K})$. Let the optimum of (39) be $\bar{x}$ with $K=\bar{K}$. One has $v(\bar{x})=\tilde{\gamma}$ and $\dot{v}(\bar{x}, \bar{\xi}, \bar{K})=0$. Thus, it yields

$$
\begin{aligned}
0 \quad & \dot{(23)} \\
\stackrel{(23)}{=} & \tilde{\psi}(\bar{x}, \xi, \bar{K}, \tilde{e}, s(x)) \\
\stackrel{(31),(32)}{=} & (*)^{T} \tilde{\Psi}(\bar{\delta}, \xi, \bar{K}, \tilde{e}, \bar{S}) \phi\left(n_{\bar{x}}, d_{\psi}\right) \\
\stackrel{(38)}{=} & (*)^{T} \bar{\Gamma}(\bar{\delta}, \xi, \bar{K}, \tilde{e}, \bar{S}) \phi\left(n_{\bar{x}}, d_{\psi}\right) .
\end{aligned}
$$

Let us observe that $\bar{\Gamma}$ is semidefinite, one has $\phi\left(n_{\bar{x}}, d_{\psi}\right)$ is in the null space of $\bar{\Gamma}$.

(Necessity) If $\phi\left(n_{\bar{x}}, d_{\psi}\right) \in \operatorname{ker}(\bar{\Gamma})$, it directly follows that

$$
\begin{aligned}
0 \stackrel{(31),(32)}{=} & (*)^{T} \tilde{\Psi}(\bar{\delta}, \xi, \bar{K}, \tilde{e}, \bar{S}) \phi\left(n_{\bar{x}}, d_{\psi}\right) \\
\stackrel{(23)}{=} & \tilde{\psi}(\bar{x}, \xi, \bar{K}, \tilde{e}, s(x)) \\
= & \dot{v}(\bar{x}, \xi, \bar{K}) \\
& +\frac{\phi_{1}}{1+\phi_{2} \tilde{\gamma}}(\tilde{\gamma}-v(\bar{x}))\left((*)^{T} \bar{S} \phi\left(n_{\bar{x}}, d_{s}\right)\right) .
\end{aligned}
$$

Then, taking into account that $\dot{v}(\bar{x}, \bar{\xi}, \bar{K})=0$ and $S>0$, one has $v(\bar{x}, \bar{\xi})=\tilde{\gamma}$. It further implies that $\gamma \leq v(\bar{x}, \bar{\xi})=\tilde{\gamma}$, while according to the definition of $\tilde{\gamma}$, it is a lower bound of $\gamma$. Thus, one has $\tilde{\gamma}=\gamma$, which completes this proof.

\section{Variable Lyapunov Functions}

In this subsection, the case of variable Lyapunov functions is considered. First, let us observe that problem (22) becomes a trilinear inequality with a variable Lyapunov function $v(x)$, which indicates that only a suboptimal solution can be obtained for (11). In addition, the measure $\rho$ in problem (11) is often selected as

$$
\rho(\mathcal{V}(c))=\operatorname{vol}(\mathcal{V}(\gamma))
$$

where $\operatorname{vol}(\mathcal{V}(\gamma))$ denotes the volume of $\mathcal{V}(\gamma)$, thus paving a way to search for a variable polynomial Lyapunov function and controller with the largest volume of $\mathcal{V}(\gamma)$. Nevertheless, choosing the volume as a measure of $\mathcal{V}(\gamma)$ makes the cost function of problem (11) non-convex, not to mention that the volume of $\mathcal{V}(\gamma)$ cannot be described by an explicit mathematical expression. In order to handle this issue, a typical way adopted in the literature is to approximate the volume of $\mathcal{V}(\gamma)$ by defining

$$
\omega=\max \frac{\gamma^{n}}{\operatorname{det}(V)}, \operatorname{vol}(\mathcal{V}(\gamma)) \propto \omega
$$

where $V$ is the SMR matrix of $v(x)$ in (30) and $\operatorname{vol}(\mathcal{V}(\gamma))$ is proportional to $\omega$. Based on this definition, a linear approximation of $\operatorname{vol}(\mathcal{V}(\gamma))$ can be proposed as

$$
\operatorname{vol}(\mathcal{V}(\gamma)) \approx \frac{\gamma}{\operatorname{trace}(V)}
$$


The idea is that minimizing the sum of the eigenvalues of matrix $V$ approximately minimizes $\operatorname{det}(V)$. For details, please find [24] for the case of quadratic Lyapunov functions.

Another possible way for coping with the variable Lyapunov functions is to enlarge $\mathcal{V}$ by employing a set of selected geometric shapes [17], [24]. In specific, one could solve the following optimization problem:

$$
\begin{aligned}
& \tilde{\mu}=\sup _{v, \epsilon} \epsilon \\
& \text { s.t. }\left\{\begin{array}{l}
\mathcal{G}(\epsilon) \subseteq \mathcal{V}(\gamma) \\
(8)-(10) \text { hold } \\
K \in \mathcal{K}, \xi_{i} \in \Xi, \forall i=1, \ldots, r .
\end{array}\right.
\end{aligned}
$$

where

$$
\mathcal{G}(\epsilon)=\left\{x \in \mathbb{R}^{n}: \mathscr{G}(x) \leq \epsilon\right\}
$$

and $\mathscr{G}(x)$ is a selected polynomial, e.g., let $\mathscr{G}(x)=\|x\|^{2}$, then the corresponding sublevel sets of $\mathcal{G}(\epsilon)$ are in a spherical shape. Similar to Theorem 1, we propose the following optimization to get a lower bound of $\rho(\mathcal{V}(c))$ :

$$
\begin{aligned}
& \bar{\mu}=\sup _{v, \epsilon} \epsilon \\
& \text { s.t. }\left\{\begin{array}{c}
\tilde{s} \in \mathcal{P}^{\mathrm{SOS}} \text { and }(\gamma-v)-\tilde{s}(\epsilon-\mathscr{G}) \in \mathcal{P}^{\mathrm{SOS}} \\
s \in \mathcal{P}_{0}^{\mathrm{SOS}} \text { and }-\psi(x, K, c, s(x)) \in \mathcal{P}_{0}^{\mathrm{SOS}} \\
\forall x \in \mathcal{V}(c) \backslash\{0\} \\
K \in \mathcal{K}, \xi_{i} \in \Xi, \forall i=1, \ldots, r .
\end{array}\right.
\end{aligned}
$$

In order to cope with (47), one typical way is by iterating among $K, s(x)$ (using the technique for the fixed Lyapunov functions) and $v(x)$. A suboptimal solution can be obtained by using existing dedicated softwares, as shown by the numerical examples in the next section [17].

\section{EXAMPLES}

To illustrate the proposed approach, two deliberately simple examples are given. Computation is carried out by a standard laptop with the Intel Core i7-4712MQ processor.

\section{A. Example 1}

Consider a 2-dimensional non-polynomial system described by

$$
\left\{\begin{array}{l}
\dot{x}_{1}=-x_{1}+x_{2}-x_{1}^{2}-5 x_{2}^{3}-\sin \left(x_{1}\right)+u_{1} \\
\dot{x}_{2}=1-2 x_{2}-4 x_{1}^{2}-e^{x_{2}}+u_{2}
\end{array}\right.
$$

with $g_{1}=g_{2}=1, \zeta_{1}=\sin \left(x_{1}\right), \zeta_{2}=1-e^{x_{2}}, h(x)=$ $\left(x_{1}, x_{2}\right)^{T}$ and $\mathcal{K}=\left\{K: K_{i j} \in[-1,1]\right\}$. Let us revisit that $d_{u}$ is the degree of $u$, we start with a fixed Lyapunov function $v_{0}(x)=x_{1}^{2}+x_{2}^{2}$ and set the truncation degree $k=5$. The bounds $\underline{\tau}_{i}$ and $\bar{\tau}_{i}$ in (7) can be selected as

$$
\begin{aligned}
& \underline{\tau}_{1}=-\sigma, \bar{\tau}_{1}=\sigma, \sigma= \begin{cases}\sin \sqrt{c}, & \text { if } \sqrt{c} \leq \pi / 2 \\
1, & \text { otherwise, }\end{cases} \\
& \underline{\tau}_{2}=-e^{\sqrt{c}}, \bar{\tau}_{2}=-e^{-\sqrt{c}} .
\end{aligned}
$$

By solving the generalized eigenvalue problem in (36), we provide the lower bounds $\tilde{\gamma}$ corresponding to the controllers with different degrees as shown in Tab. 1. and Fig. 1.

From Fig. 1, it shows that by using the polynomial controller $u$, the largest estimate of the DA has been greatly
TABLE I

THE LOWER BOUND $\tilde{\gamma}$ FOR SOME VALUES OF $d_{u}$ AND THE CORRESPONDING COMPUTATIONAL TIME $t_{c}$.

\begin{tabular}{cccccc}
\hline$d_{u}$ & 0 & 1 & 2 & 3 & 4 \\
\hline$\tilde{\gamma}$ & 0.8044 & 1.818 & 3.3012 & 133.44 & 352.73 \\
$t_{c}[\mathrm{~s}]$ & 5.757 & 6.138 & 6.308 & 5.324 & 7.864 \\
\hline
\end{tabular}

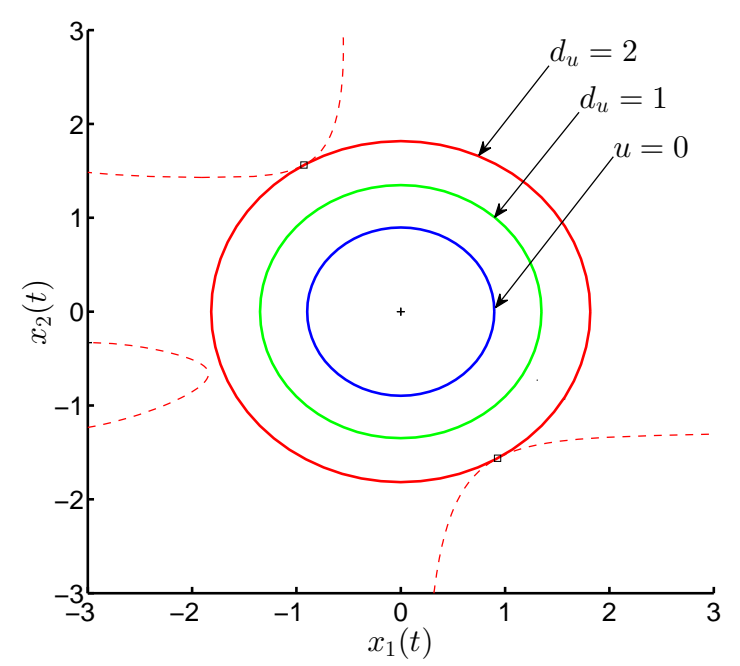

Fig. 1. Example 1: The solid lines indicate the bounds of the largest estimate of the the DA. The blue line, the green line and the red line stand for the cases $u=0, d_{u}=1$, and $d_{u}=2$ respectively. The dashed line indicates $\dot{v}(x, \bar{\xi}, \bar{K})=0$ corresponding to the case $d_{u}=2$.

expanded. The optimal control inputs for the cases $d_{u}=1$ and $d_{u}=2$ are $\bar{u}_{d 1}=\left(-x_{1}+0.2369 x_{2}, 0.2369 x_{1}-\right.$ $\left.x_{2}\right)^{T}$ and $\bar{u}_{d 2}=\left(-x_{1}+x_{2}+0.5003 x_{1}^{2}+0.9508 x_{1} x_{2}-\right.$ $\left.0.9151 x_{2}^{2}, x_{1}-x_{2}+0.9508 x_{1}^{2}-0.9151 x_{1} x_{2}+0.1778 x_{2}^{2}\right)^{T}$ respectively. In addition, it also indicates that the tightness of the lower bound is established with $\tilde{\gamma}=\gamma(\bar{K})$ at points $\bar{x}_{1}=(0.9274,-1.563)$ and $\bar{x}_{2}=(-0.9279,1.562)$ where $\bar{x} \in \mathcal{T}$ from Theorem 3 by solving LMIs.

Next, we use the variable Lyapunov function to further expand the largest estimate of the DA, based on the result we achieved. By using a linear approximation of $\operatorname{vol}(\mathcal{V}(\gamma))$, an optimal quadratic Lyapunov function is found at the minimum of trace $(V)$ as shown in Fig. 2. The result shows that $\operatorname{trace}_{\min }(V)=0.42561$ and the corresponding optimal Lyapunov function is $\bar{v}(x)=0.1576 x_{1}^{2}-0.1242 x_{1} x_{2}+$ $0.268 x_{2}^{2}$. Then, we fix this Lyapunov function and continue to find a controller with $d_{u}=2$ to enlarge the largest estimate of the DA. Finally, an optimal controller is found: $\tilde{u}_{d 2}=\left(-x_{1}+x_{2}+0.1347 x_{1}^{2}+0.2857 x_{1} x_{2}+0.4426 x_{2}^{2}, x_{1}-\right.$ $\left.x_{2}+0.5819 x_{1}^{2}+0.6139 x_{1} x_{2}+0.3343 x_{2}^{2}\right)^{T}$. 


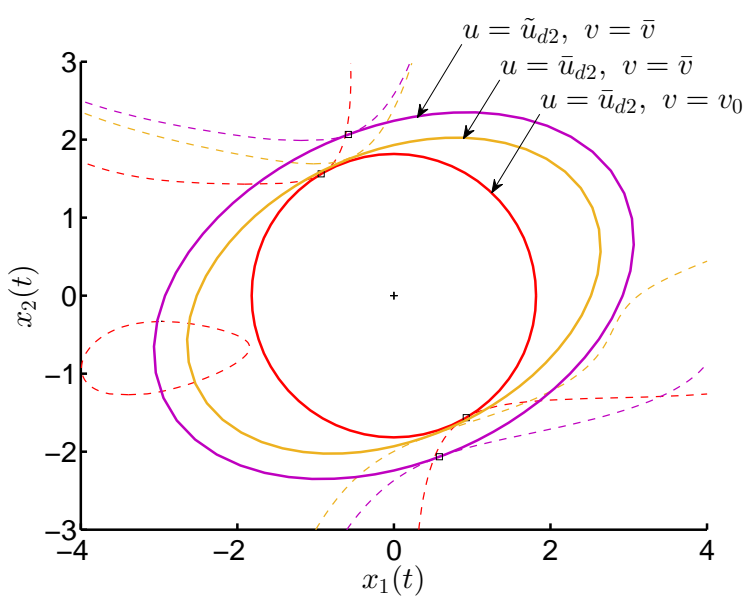

Fig. 2. Example 1: The solid lines indicate the bounds of the largest estimate of the DA. The red line and the yellow line stand for the fixed Lyapunov function and the optimal one with $d_{v}=2$ respectively. The purple line indicates the further enlarged result by fixing the found optimal Lyapunov function and by using the proposed controller with $d_{u}=2$. The dashed line of corresponding color indicates $\dot{v}(x, \bar{\xi}, \bar{K})=0$ correspondingly.

\section{B. Example 2}

For this case, we consider the non-polynomial system

$$
\left\{\begin{array}{l}
\dot{x}_{1}=x_{2}+x_{3}^{2} \\
\dot{x}_{2}=x_{3}-x_{1}^{2}-x_{1} \sin \left(x_{1}\right)+u_{1} \\
\dot{x}_{3}=-x_{1}-2 x_{2}-x_{3}+x_{2}^{3}+\ln \left(\frac{1+x_{3}}{1-x_{3}}\right)^{\frac{1}{10}}+u_{2} \\
y_{1}=x_{1}-x_{2} \\
y_{2}=x_{2}-x_{3}
\end{array}\right.
$$

with $g_{1}=x_{1}, g_{2}=0.1, \zeta_{1}=\sin x_{1}, \zeta_{2}=\ln \left(\frac{1+x_{3}}{1-x_{3}}\right), h(x)=$ $\left(x_{1}-x_{2}, x_{2}-x_{3}\right)^{T}$ and $\mathcal{K}=\left\{K: K_{i j} \in[-1,1]\right\}$. We exploit a fixed polynomial Lyapunov function $v_{0}(x)=2 x_{1}^{4}+$ $2 x_{1}^{2} x_{2}^{2}+5 x_{1}^{2}+10 x_{1} x_{2}+2 x_{1} x_{3}+10 x_{2}^{2}+8 x_{2}^{4}+6 x_{2} x_{3}+4 x_{3}^{2}+$ $3 x_{3}^{4}$ and set the truncation degree as $k=3$. By solving (36), we search a polynomial controller with $d_{u}=2$ and establish the tightness of the lower bounds $\tilde{\gamma}$ corresponding to this controllers as shown in Fig. 3.

By using Theorem 2, we find $\tilde{\gamma}=0.9227$ and the corresponding optimal control inputs is $\bar{u}=$ $\left(-0.3432 y_{1}-0.7632 y_{2}+0.9933 y_{1}^{2}+0.1599 y_{1} y_{2}-\right.$ $0.8012 y_{2}^{2}, 0.05074 y_{1}+0.8147 y_{2}-0.4789 y_{1}^{2}+0.9501 y_{1} y_{2}-$ $\left.0.9171 y_{2}^{2}\right)^{T}$. Furthermore, the result also shows that the tightness of the lower bound is established with $\tilde{\gamma}=\gamma(\bar{K})$ at points $(0.4193,-0.344,-0.05943)$, $(-0.401,0.02484,-0.134)$ and $(-0.2056,0.32,0.124)$.

\section{CONCLUSION}

For a class of non-polynomial systems, a novel approach is proposed that a polynomial output feedback controller can be computed to maximize the largest estimate of the DA. By introducing a new class of SMR for the set of local SOS and a transformation of power vector, the lower bound of the largest estimate of the DA can be calculated via a quasiconvex optimization consisting of a generalized eigenvalue problem for a fixed Lyapunov function. Furthermore, the

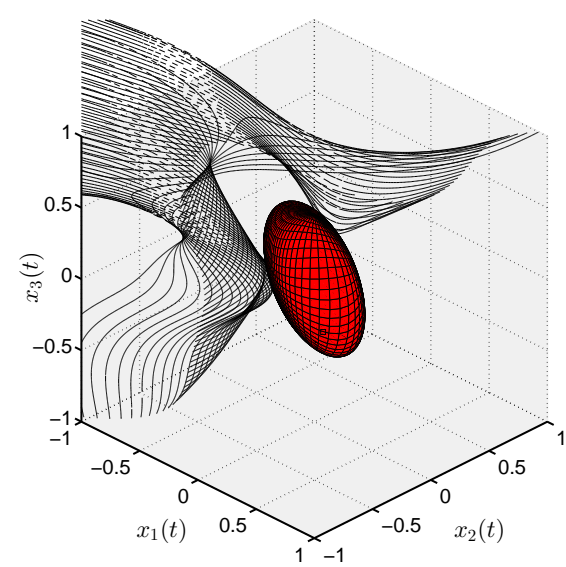

Fig. 3. Example 2: The red spheroid indicates the bounds of the largest estimate of DA with $d_{u}=2$. The black solid line indicates $\dot{v}(x, \bar{\xi}, \bar{K})=0$.

tightness of the found lower bound can be established by a necessary and sufficient condition. Lastly, several methods have been discussed for the case of the variable Lyapunov functions.

Future efforts will focus on designing a less-conservative convex approach to approximate $\operatorname{vol}(\mathcal{V}(\gamma))$ and enlarge the lower bound of $\gamma$ for the case of variable Lyapunov functions. In addition, we are interested to compare this approach with other stability verification methods, like reachability analysis methods and contraction theory methods [25], [26].

\section{ACKOWLEDGEMENTS}

The authors would like to thank the Editors and the Reviewers for their valuable comments. This work is supported by the German Research Foundation (AL 1185/2-1).

\section{APPENDIX}

\section{A. Proof of Lemma 4}

By using the Kronecker product, $v(x)$ and $v(x) s(x)$ can be expressed by a common power vector of SMR. In specific, we rewrite $\varsigma(x)$ as

$$
\begin{aligned}
\varsigma(x)= & \left((*)^{T}\left[\begin{array}{cc}
\varphi_{1} & 0 \\
0 & \varphi_{2} V
\end{array}\right]\left[\begin{array}{c}
1 \\
\phi\left(n, d_{\psi}\right)
\end{array}\right]\right) \\
& \cdot(*)^{T} S \phi\left(n, d_{s}\right) \\
= & (*)^{T}\left(\left[\begin{array}{cc}
\varphi_{1} & 0 \\
0 & \varphi_{2} V
\end{array}\right] \otimes S\right) \\
& \cdot\left(\left[\begin{array}{c}
1 \\
\phi\left(n, d_{\psi}\right)
\end{array}\right] \otimes \phi\left(n, d_{s}\right)\right) \\
= & \phi\left(n, d_{\psi}\right)^{T}\left((*)^{T}\left(\left[\begin{array}{cc}
\varphi_{1} & 0 \\
0 & \varphi_{2} V
\end{array}\right]\right) H\right) \\
& \cdot \phi\left(n, d_{\psi}\right) \\
= & (*)^{T} \Lambda(S) \phi\left(n, d_{\psi}\right) .
\end{aligned}
$$




\section{B. Proof of Proposition 1}

Comparing the pursuits of $\gamma$ and $c_{k}$ given by (12) and (22), the objective function are the same while the constraints are different. In particular, for the case of $\gamma$, it is required that

$$
\forall x \in \mathcal{V}(c) \backslash\{0\}: \forall \xi_{i} \in \Xi: \dot{v}\left(x, K, \xi_{i}\right)<0 .
$$

In contrast, for the case of $c_{k}$ with $k \rightarrow \infty$, it requires

$\forall \xi_{i} \in \operatorname{ver}(\Xi), \forall x \in \mathcal{V}(c) \backslash\{0\}: r(x)+q(x)^{T} \xi+e(x, K)<0$

From (26), we have that, for a specific $\bar{K}$ and for all $x \in$ $\mathcal{V}(c) \backslash\{0\}$, there exists $\bar{\xi}_{i} \in \Xi, i=1, \ldots, r$, such that

$$
r(x)+q(x)^{T} \xi+e(x, \bar{K})-\dot{v}(x, \bar{K}, \bar{\xi})=q(x)^{T} \xi-q(x)^{T} \bar{\xi}
$$

where $\bar{\xi}=\left(\bar{\xi}_{1}, \ldots, \bar{\xi}_{r}\right)^{T}$. Taking into account (1) and the definition of $\theta$, it yields that

$$
\left|r(x)+q(x)^{T} \xi+e(x, \bar{K})-\dot{v}(x, \bar{K})\right| \leq 2 \theta \sqrt{r} \sup \|q(x)\|
$$

for all $x \in \mathcal{V}(c) \backslash\{0\}$. In addition, from (18)-(21), one has

$$
q(x)=\frac{\bar{q}(x)}{k !}
$$

where $\bar{q}(x)$ is a vector of polynomials. Based on this, for any finite scalar $c$ and $x \in \mathcal{V}(c) \backslash\{0\}$, one has that

$$
\begin{aligned}
& \lim _{k \rightarrow \infty}\left|r(x)+q(x)^{T} \xi+e(x, \bar{K})-\dot{v}(x, \bar{K}, \bar{\xi})\right| \\
\leq & \lim _{k \rightarrow \infty} \sup \|q(x)\| \\
\leq & 2 \theta \sqrt{r} \lim _{k \rightarrow \infty} \frac{1}{k !} \sup \|\bar{q}(x)\| .
\end{aligned}
$$

Observe that $\|\bar{q}(x)\|$ is a vector of polynomial which is finite over the bounded domain $\mathcal{V}(c)$, one has that

$$
\lim _{k \rightarrow \infty}\left|r(x)+q(x)^{T} \xi+e(x, \bar{K})-\dot{v}(x, \bar{K}, \bar{\xi})\right|=0 .
$$

Therefore, the difference between $\gamma$ and $c_{k}$ vanishes as $k$ tends to infinity.

\section{REFERENCES}

[1] H. K. Khalil and J. W. Grizzle. Nonlinear systems. Prentice hall Upper Saddle River, 2002.

[2] D. Henrion and J.-B. Lasserre. GloptiPoly: Global optimization over polynomials with MATLAB and SeDuMi. ACM Transactions on Mathematical Software, 29(2):165-194, 2003.

[3] P. A. Parrilo. Structured semidefinite programs and semialgebraic geometry methods in robustness and optimization. $\mathrm{PhD}$ thesis, California Institute of Technology, 2000.

[4] G. Chesi. LMI techniques for optimization over polynomials in control: a survey. IEEE Transactions on Automatic Control, 55(11):2500$2510,2010$.

[5] D. Henrion and M. Korda. Convex computation of the region of attraction of polynomial control systems. IEEE Transactions on Automatic Control, 59(2):297-312, 2014.

[6] A. Papachristodoulou and S. Prajna. A tutorial on sum of squares techniques for systems analysis. In Proceedings of the American Control Conference, pages 2686-2700, 2005.

[7] U. Topcu, A. Packard, and P. Seiler. Local stability analysis using simulations and sum-of-squares programming. Automatica, 44(10):2669 - 2675, 2008.

[8] V. Powers and T. Wörmann. An algorithm for sums of squares of real polynomials. Journal of Pure and Applied Algebra, 127(1):99-104, 1998.

[9] G. Chesi. Computing output feedback controllers to enlarge the domain of attraction in polynomial systems. IEEE Transactions on Automatic Control, 49(10):1846-1853, 2004.
[10] S. Mastellone, P. F. Hokayem, C. T. Abdallah, and P. Dorato. Nonlinear stability analysis for non-polynomial systems. In Proceedings of the American Control Conference, pages 1725-1730, 2004.

[11] G. Hexner. Stability of non-polynomial systems using differential inclusions and polynomial Lyapunov functions. In Proceedings of Conference on Decision and Control, pages 2946-2951, 2012.

[12] A. Papachristodoulou and S. Prajna. Analysis of non-polynomial systems using the sum of squares decomposition. In Positive polynomials in control, volume 312 of Lecture Notes in Control and Information Science, pages 23-43. Springer, 2005.

[13] G. Chesi. Estimating the domain of attraction for non-polynomial systems via LMI optimizations. Automatica, 45(6):1536-1541, 2009.

[14] T. Gußner, M. Jost, and J. Adamy. Controller design for a class of nonlinear systems with input saturation using convex optimization. Systems \& Control Letters, 61(1):258-265, 2012.

[15] A. Saleme and B. Tibken. Nonlinear static state feedback controller design to enlarge the domain of attraction for a class of nonlinear systems. In Proceedings of the American Control Conference, pages 4074-4079, 2013

[16] M. Berz and G. Hoffstätter. Computation and application of Taylor polynomials with interval remainder bounds. Reliable Computing, 4(1):83-97, 1998

[17] Z. Jarvis-Wloszek, R. Feeley, W. Tan, K. Sun, and A. Packard. Some controls applications of sum of squares programming. In Proceedings of Conference on Decision and Control, pages 4676-4681, 2003.

[18] W. Tan and A. Packard. Stability region analysis using polynomial and composite polynomial Lyapunov functions and sum-of-squares programming. IEEE Transactions on Automatic Control, 53(2):565$570,2008$.

[19] J. Bochnak, M. Coste, and M.-F. Roy. Real algebraic geometry. Springer, 1998.

[20] K. Schmüdgen. The K-moment problem for compact semi-algebraic sets. Mathematische Annalen, 289(1):203-206, 1991.

[21] G. Stengle. A Nullstellensatz and a Positivstellensatz in semialgebraic geometry. Mathematische Annalen, 207(2):87-97, 1974.

[22] M. Putinar. Positive polynomials on compact semi-algebraic sets Indiana University Mathematics Journal, 42(3):969-984, 1993.

[23] S. Boyd, L. El Ghaoui, E. Feron, and V. Balakrishnan. Linear matrix inequalities in system and control theory. Society for industrial and applied mathematics, 1994.

[24] G. Chesi, A. Garulli, A. Tesi, and A. Vicino. LMI-based computation of optimal quadratic Lyapunov functions for odd polynomial systems. International Journal of Robust and Nonlinear Control, 15(1):35-49, 2005.

[25] M. Althoff and B. H. Krogh. Reachability analysis of nonlinear differential-algebraic systems. IEEE Transactions on Automatic Control, 59(2):371-383, 2014.

[26] D. Han and G. Chesi. Robust synchronization via homogeneous parameter-dependent polynomial contraction matrix. IEEE Transactions on Circuits and Systems I: Regular Papers, 61(10):2931-2940, 2014. 